\title{
Prevalence of Ciprofloxacin and Nalidixic Acid Resistant Salmonella enterica serovar Typhi in Bangladesh
}

\author{
Shirin Afroj ${ }^{1}$, Mohammad Ilias ${ }^{1}$, Maksuda Islam ${ }^{2}$, and Samir K Saha ${ }^{2 *}$ \\ ${ }^{I}$ Department of Microbiology, University of Dhaka, Dhaka-1000, Bangladesh. ${ }^{2}$ Department of Microbiology, Dhaka Shishu (Children) Hospital, \\ Dhaka-1207, Bangladesh
}

(Received 3 January 2011; Accepted 9 April 2011)

\begin{abstract}
A total of 1,059 Salmonella enterica serovar Typhi were isolated from blood samples during January 2006 to October 2007 from urban rural facilities in Dhaka, Bangladesh, of which $980(\mathbf{9 2 . 5 \%})$ isolates were nalidixic acid resistant. The minimum inhibitory concentrations (MIC) of ciprofloxacin (CIP) were determined for 127 nalidixic acid resistant Salmonella enterica serovar Typhi (NARST) strains (every fifth) isolated during 2006. Nine isolates were found to be resistant against CIP (3\%) with high MIC $(12->32 \mu \mathrm{g} / \mathrm{mL})$. Only four isolates were found to be sensitive (MIC $<0.125 \mu \mathrm{g} / \mathrm{mL}$ ), whereas most of the isolates $(\mathrm{N}=113)$ showed reduced susceptibility (MIC $0.125-2 \mu \mathrm{g} / \mathrm{mL}$ ) to CIP. All these isolates were subjected to molecular typing by multiplex PCR on VNTR (variable number tandem repeats) loci, which revealed eight different VNTR patterns. Almost all CIP resistant strains had similar genetic organization, identical to the most common VNTR type. Restriction fragment length polymorphism (RFLP) analysis of the gyrA gene revealed point mutations at Ser-83 and Asp87 in all CIP resistant strains.
\end{abstract}

Key words: Salmonella enterica serovar Typhi, fluoroquinolone resistant, VNTR

\section{Introduction}

Diseases caused by Salmonella enterica serovars are especially prevalent in developing countries. Typhoid fever is sometimes a fatal infection to adults and children that causes bacteremia and imflammatory destruction of the intestine and other organs. This requires an urgent treatment by the administration of appropriate antibiotics. Emergence of multidrug-resistant (MDR) S. enterica serovar Typhi strains in Asia in the beginning of 1990s, led to the widespread use of fluoroquinolones for treating enteric fever ${ }^{1-2}$. MDR typhoid fever endemic started in Bangladesh in 1990s, which reached to peak in 1994, and then declined and re-emerged in 2001 and $2002^{3}$. During the last decade, several treatment failures of S. typhi strains with decreased susceptibility to ciprofloxacin ${ }^{3-}$ ${ }^{5}$ have been reported and some studies also confirmed the presence of fluoroquinolone resistant $S$. enterica serovar Typhi strains $^{6-8}$. Antibiotic-resistant Salmonella strains pose a significant threat to the development of reliable therapies. $S$. enterica serovar Typhi with resistance to nalidixic acid and decreased susceptibilities or resistance to fluoroquinolones have been increasingly reported in several countries including Bangladesh $^{7,9-13}$. Such strains have also been reported from other parts of the world, most of them have travel history to Asian countries particularly South East Asia ${ }^{6,14}$.

The mechanisms of fluoroquinolone resistance have been well studied. Single point mutation in the quinolone resistance- determining region (QRDR) of the gyrA gene in Salmonella usually leads to resistance against nalidixic acid and to decreased susceptibility to ciprofloxacin ${ }^{7,15-16}$. Turner et al. ${ }^{17}$ reported that two substitutions in gyrA (Ser-83 $\rightarrow$ Phe or Tyr and Asp-87 $\rightarrow$ Asn) and one in $\operatorname{parC}$ (Glu-84 $\rightarrow$ Lys) confer complete fluoroquinolone resistance in $S$. typhi. Emergence of these resistant strains are associated with treatment failure or delayed response to cipropfloxacin ${ }^{9,18}$.

There are few reports on the occurrence of typhoid by absolute fluoroquinolone resistant strain in Bangladesh. Here, we report nine cases of typhoid fever in Dhaka, Bangladesh caused by $S$. enterica serovar Typhi with complete resistance to ciprofloxacin during 2006.

\section{Materials and Methods \\ Specimen collection and identification of Salmonella enterica serovar Typhi}

The patients were selected for specimen collection based on the clinical manifestation of typhoid fever diagnosed by physician from different sites at Dhaka City, Bangladesh. Blood samples were collected along with patient's demographic data from all age group of typhoid patients during 2006 to October 2007, as a part of routine diagnosis.

Blood cultures were performed by the lysis centrifugation method as described in our previous report ${ }^{19}$. Positive cultures were 
subsequently plated on blood, choocolate and MacConkey agar plates. Colonies of Salmonella enterica serovar Typhi were identified by standard biochemical and serological procedures ${ }^{20}$.

Determination of antibiotic sensitivity and MIC of ciprofloxacin

Antibiotic susceptibility testing was performed by disk diffusion method using discs (Oxoid, UK) containing ampicillin $(10 \mu \mathrm{g})$, ceftriaxone $(30 \mu \mathrm{g})$, cotrimoxazole $(25 \mu \mathrm{g})$, chloramphenicol (30 $\mu \mathrm{g})$, ceftazidime $(30 \mu \mathrm{g})$, ciprofloxacin $(5 \mu \mathrm{g})$ and nalidixic acid (30 $\mu \mathrm{g})$. Based on the date of isolation, every fifth nalidixic acid resistant $S$. enterica serovar Typhi (NARST) isolated during the year 2006 were selected to determine the MIC of ciprofloxacin (CIP) by E-strip. All results were interpreted according to Clinical and Laboratory Standards Institute (CLSI) guidelines ${ }^{21}$ and were analysed by SPSS version 11.5. The trend of multidrug resistance, which is defined as resistance to ampicillin, chloramphenicol and co-trimoxazole was also analysed during 2006 to October 2007.

\section{Multiplex PCR on VNTR loci}

All the isolATES whose MIC was determined by E-strip test were subjected to molecular typing by multiplex PCR on variable number tandem repeats (VNTR) using primers flanking three VNTR loci (TR1, TR2 and TR3) (Table 1), as described by Liu et $a l .{ }^{22}$. In brief, each $25 \mu \mathrm{L}$ of reaction mixture contained $1.5 \mu \mathrm{L}$ of the bacterial lysate suspension and $10 \mathrm{pmol}$ each of the forward and reverse primers for TR1 and TR3, as well as 12.5 pmol each of the corresponding primers for TR2, in addition to $22 \mu \mathrm{L}$ of the Taq PCR mastermix (QIAGEN GmbH, Hilden, Germany). After initial denaturation at $94^{\circ} \mathrm{C}$ for $5 \mathrm{~min}$, the $\mathrm{PCR}$ reaction was run for 35 cycles at $94^{\circ} \mathrm{C}$ for $30 \mathrm{~s}, 55^{\circ} \mathrm{C}$ for $30 \mathrm{~s}$, and $72^{\circ} \mathrm{C}$ for $1 \mathrm{~min}$, followed by a final extension at $72^{\circ} \mathrm{C}$ for $7 \mathrm{~min}$. The $\mathrm{PCR}$ products, along with a 100-bp DNA marker (Invitrogen, USA), were subjected to electrophoresis on a $2 \%$ agarose gel. In every case, positive and negative controls were run simultaneously.

Restriction fragment length polymorphism (RFLP) analysis

PCR-RFLP was performed to detect common mutations related to fluoroquinolone resistance at the codon Ser-83 and Asp-87 of gyrA following the procedure described by Hirose et al. ${ }^{23}$. PCR was performed with the primers, gyrA-F, 5' TGT CCG AGA TGG CCT GAA GC 3' and gyrA-Hinfl-as, 5'ATG TAA CGC AGC GAG AAT GGC TGC GCC ATA CGAACG CTG GAG 3'. The 195-bp amplified product contains two Hinfl restriction sites at the codon corresponding to Ser-83 and Asp-87 of gyrA. Restriction digestions were performed in a total of $15 \mu \mathrm{L}$ reaction mixture containing $8 \mu \mathrm{L}$ of the above-mentioned PCR product, $1.5 \mu \mathrm{L}$ of $10 \mathrm{x}$ digestion buffer and $10 \mathrm{U}$ of Hinfl for overnight at $37^{\circ} \mathrm{C}$. The restriction digests were electrophoresed onto a $3 \%$ agarose gel. Hinfl-digested PCR products of gyrA gene from a known nalidixic acid sensitive $S$. enterica serover Typhi strain, and the undigested product from known ciprofloxacin resistant strain, were run as controls.

\section{Results}

During the period of the study (2006 to October 2007), a total of 1,059 Salmonella enterica serovar Typhi were isolated. The majority (57\%; 605 out of 1059) of the S. enterica isolates were isolated in the year 2006. A significant higher proportion of cases (43\%) occurred in patients younger than 5 years of age than in those 5 years of age $(9 \%)$ or older. The highest rate $(13 \%)$ of $S$. enterica serovar Typhi isolation was in the second year of life (Fig. 1).

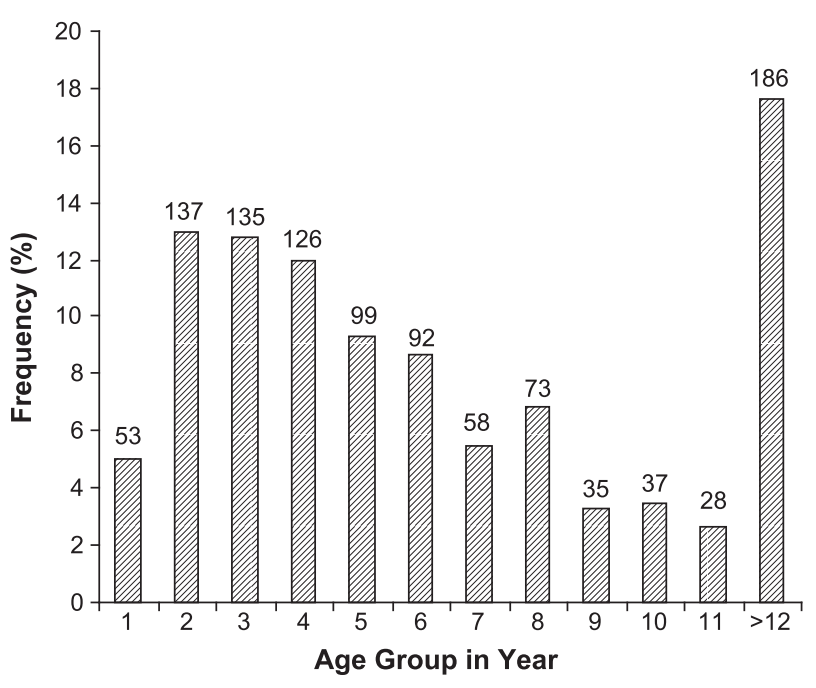

Figure 1. Age distribution of typhoid fever cases caused by $S$. enterica serovar Typhi identified at a diagnostic referral center and Dhaka Shishu Hospital, Bangladesh during 2006-2007 (n =1059). Numbers above the bars show the total numbers of isolates

Table 1. PCR primers flanking the three VNTR loci of S. enterica serovar Typhi ${ }^{22}$.

\begin{tabular}{llll}
\hline Primer & Nucleotide positions & Sequence $\left(5^{\prime}-3^{\prime}\right)$ & VNTR sequence \\
\hline TR1F1 & $2017115-2017136$ & AGA ACC AGC AAT GCG CCA ACG A & (AGAAGAA) 12 \\
TR1R1 & $2017354-2017375$ & CAA GAA GTG CGC ATA CTA CAC C & \\
TR2F1 & $2556810-2556831$ & CCC TGT TTT TCG TGC TGA TAC G & $($ CCAGTTCC) 27 \\
TR2R2 & $2557299-2557320$ & CAG AGG ATA TCG CAA CAA TCG G & $($ CGCGGGGATCGGTTT \\
TR3F1 & $2926145-2926166$ & CGA AGG CGG AAA AAA CGT CCT G & ATCCCCGCTGG) \\
& & &
\end{tabular}


Antibiotic susceptibility patterns of 1,059 S. enterica serovar Typhi were analysed for seven antibiotics. Among them only 52 $(4.9 \%)$ isolates were sensitive to all antimicrobial agents tested. Although no resistance to cefriaxone (CRO) or ceftazidime (CAZ) was observed, 92.5\% isolates were nalidixic acid (NA) resistant. Multidrug resistance (MDR) was noted in around 56\% (593 out of 1059) of the isolates. Moreover, MDR strains were more likely to be resistant to NA, in comparison to sensitive strains $(96.7 \%$ vs $3.3 \%)$.

The results of molecular typing and MIC for ciprofloxacin are presented in Table 2 and Figure 2 respectively. Although not considered resistance by current CLSI standard breakpoints, 89\% (113 out of 127) isolates demonstrated a "reduced susceptibility" to ciprofloxacin (MIC value 0.125 to $2 \mu \mathrm{g} / \mathrm{mL}$ ) and 9 isolates were completely resistant to CIP (MIC value 12 - >32 $\mu \mathrm{g} / \mathrm{mL}$ ) (Fig. 2).

Table 2. Different VNTR types and their corresponding amplicon size.

\begin{tabular}{lcc}
\hline VNTR Type & Number of isolates & Amplicon size (bp) \\
\hline A & 93 & $\sim 200,>600$ \\
B & 14 & $>200,>600$ \\
C & 12 & $>300, \sim 500$ \\
D & 2 & $\sim 150,>200, \sim 600$ \\
E & 2 & $>250,>300, \sim 600$ \\
F & 2 & $>250, \sim 600$ \\
G & 1 & $200,<400,>600$ \\
H & 1 & $>200$ \\
\hline
\end{tabular}

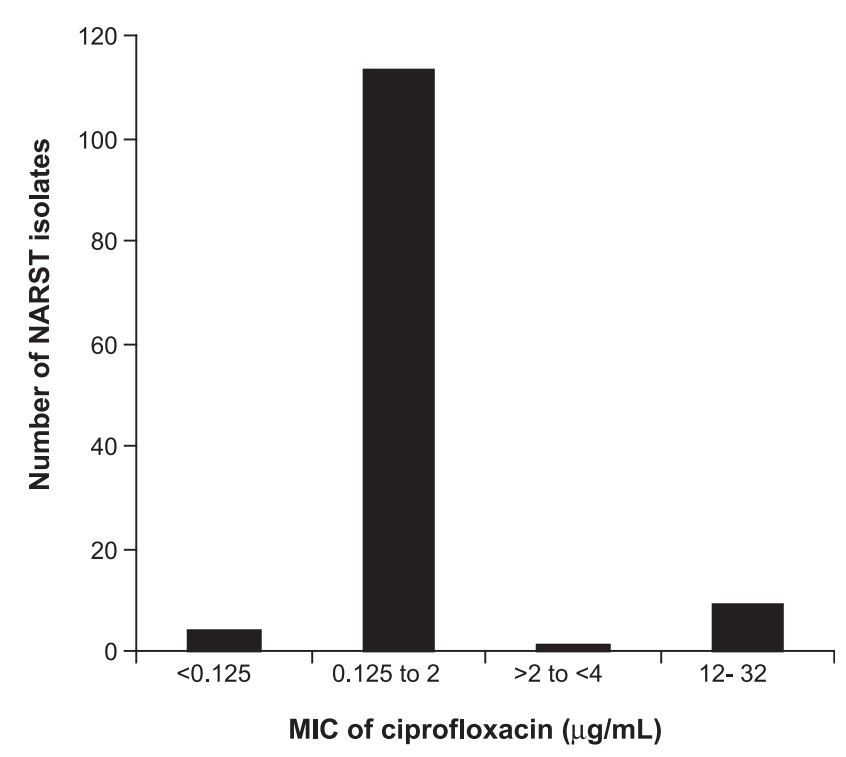

Figure 2. MIC of ciprofloxacin of some nalidixic acid resistant Salmonella enterica serovar Typhi $(n=127)$ isolated in 2006. MIC value $<0.125 \mu \mathrm{g} / \mathrm{mL}$, Sensitive; MIC value 0.125 to $2 \mu \mathrm{g}$ / $\mathrm{ml}$, decreased susceptibility; MIC value $>2$ to $<4 \mu \mathrm{g} / \mathrm{mL}$, intermediate; MIC value 12 - >32 $\mu \mathrm{g} / \mathrm{mL}$, resistant
All the NARST isolates were subjected to molecular typing on VNTR loci through multiplex PCR. A total of 8 VNTR types (type A to type $\mathrm{H}$ ) based on the number and size of bands were identified among 127 isolates (Table 2). Most of the isolates $73 \%(93 / 127)$ belonged to the VNTR type A and eight of the nine ciprofloxacin resistant isolates were also included in this group.

The fragments containing the gyrA QRDR of nine ciprofloxacin resistant isolates were amplified by PCR. The 195 bp PCR products of CIP resistant strains were not digested with Hinfl (Fig. 3), suggesting point mutations at both Ser-83 and Asp-87 of gyrA. In contrast, the PCR product from nalidixic acid susceptible control strain cleaved at both HinfI sites at the codon corresponding to Ser-83 and Asp-87 of gyrA (Fig. 3).

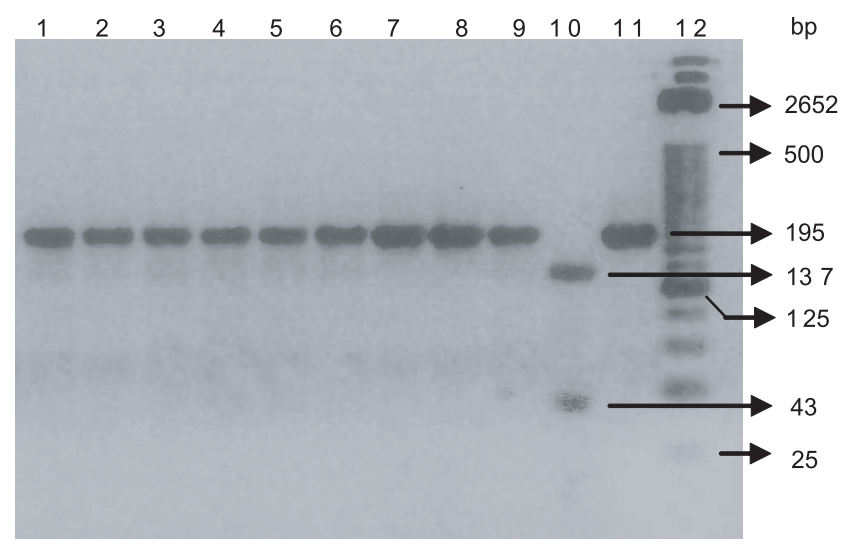

Figure 3. PCR-RFLP Pattern of S. enterica serovar Typhi based on digestion of gyrA gene (195 bp) with HinfI restriction enzyme. Lanes 1-9: Treated gyrase PCR product of Cip ${ }^{R}$ strains; Lanes 10: Treated gyrase product of $N A^{S}$ strain; Lanes 11: Non-treated gyrase PCR product of Cip ${ }^{R}$ strain; Lanes 12: 25 bp DNA Ladder (Invitrogen)

\section{Discussion}

Typhoid fever is still endemic in developing countries with fatal infection in children and occasionally in adults. In the present study the disease was found highest in young children ranged within 2-3 years. This result corroborates with our previous study in which we reported the highest incidence of the disease in children younger than 5 years of age and more than one fourth of cases occurred in the first two years of life ${ }^{24}$. The prevalence of $S$. typhi infection in younger children ( $<5$ years) has also been reported by several other groups ${ }^{25-27}$.

Using the breakpoints recommended by the CLSI ${ }^{21}$, nine typical S. enterica serovar Typhi isolates resistant to ciprofloxacin were isolated in this study. However, most of the isolates (89\%) were found with decreased susceptibility to ciprofloxacin (MICs ranged from $0.125-2.0 \mu \mathrm{g} / \mathrm{mL}$ ). The isolates with decreased ciprofloxacin susceptibility were also uniformly resistant to nalidixic acid. Recently, S. enterica serovar Typhi isolates with decreased ciprofloxacin susceptibility (MIC, $>0.125 \mu \mathrm{g} / \mathrm{mL}$ ) have become 
the subject of worldwide attention ${ }^{28}$. Although none of these isolates were phenotypically "resistant" according to the current CLSI guidelines, treatment failure in a substantial proportion of our patients treated with ciprofloxacin has been observed. The documentation of clinician as well as microbiological failure suggests a need to re-evaluate the interpretive MIC breakpoints of fluoroquinolones, since the current reference standards are unable to distinguish between fluoroquinolone susceptible isolates and isolate with reduced susceptibility ${ }^{7,29}$.

The multiplex PCR based VNTR profiling revealed eight different types of VNTR patterns, among them 93 (73\%) isolates showed two bands corresponding to $\sim 200,>600$ bp (type A). Eight out of nine ciprofloxacin resistant isolates also gave the same banding pattern as type A. As most of the isolates gave the same banding pattern like resistant strains and MIC of these strains ranged from 0.125 to $2 \mu \mathrm{g} / \mathrm{mL}$ (reduced susceptible group), therefore indicating all of these isolates are prone to resistance to fluoroquinolones.

Our previous report identified three ciprofloxacin resistant isolates in 2005, afterward we identified nine resistant strains with high MIC value $(12->32 \mu \mathrm{g} / \mathrm{mL})$ in 2006 . These results suggest that NARST with reduced susceptibility to ciprofloxacin is now endemic in Bangladesh. Only a few cases of fluoroquinolone resistant isolates have been reported up to now, but the increasing number of isolates resistant to nalidixic acid with decreased susceptibility to ciprofloxacin is a matter of concern. There is also an urgent need to reevaluate fluoroquinolones breakpoints for S. enterica serovar Typhi.

More recently, Salmonella spp. isolates, exhibiting reduced susceptibility to ciprofloxacin but sensitive to nalidixic acid has been observed ${ }^{30}$. Therefore, screening method for detection of reduced susceptible group to ciprofloxacin by nalidixic acid disc diffusion need reconsideration. Until then, laboratory should include MIC method along with standard disc diffusion, particularly in endemic region.

This study has the limitation that patient information was collected retrospectively, only after confirmation that the isolates were $S$. enterica serovar Typhi. Furthermore, we could not confirm the mutation in gyr A, which contain the quinolone resistance determining region, by DNA sequencing. The emergence of highly ciprofloxacin-resistant isolates may be due to the overuse of this drug in a population with high prevalence of NARST, as predicted by Hirose et al. ${ }^{23}$. Therefore, now it is a prime concern to consider new, effective and alternative choice of drug in forthcoming days to combat against typhoid caused by ciprofloxacin resistant $S$. enterica serovar Typhi. All isolates investigated here including ciprofloxacin-resistant isolates were susceptible to ceftriaxone and azithromycin (data not shown), which might indicate that these antibiotics could still provide an appropriate therapy for typhoid fever. But the increased therapeutic cost, together with difficulties in intravenous administration can be a serious handicap in developing countries like Bangladesh.

\section{Acknowledgements}

The study was partially supported by The Child Health Research Foundation and Popular Diagnostic Centre Ltd, Bangladesh. We gratefully acknowledge these donors for their support and commitment to this investigation.

\section{References}

1. Rahman MM, Haq JA, Morshed MA, Rahman MA. 2005. Salmonella enterica serovar Typhi with decreased susceptibility to ciprofloxacinan emerging problem in Bangladesh. Int $J$ Antimicrob Agents. 25: 345-346.

2. Renuka K, Sood S, Das BK, Kapil A. 2005. High-level ciprofloxacin resistance in Salmonella enterica serotype Typhi in India. $J$ Med Microbiol. 54: 999-1000.

3. Rahman M, Siddique AK, Shoma S, Rashid H, Salam MA, Ahmed QS, Nair GB, Breiman RF. 2006. Emergence of multidrug-resistant Salmonella enterica serotype Typhi with decreased ciprofloxacin susceptibility in Bangladesh. Epidemiol Infect. 134: 433-438.

4. Launay O, Nguyen Van JC, Buu-Hoi A, Acar JF. 1997. Typhoid fever due to a Salmonella typhi strain of reduced susceptibility to fluoroquinolones. Clin Microbiol Infect. 3: 541-544.

5. Slinger R, Desjardins M, McCarthy AE, Ramotar K, Jessamine P, Guibord C, Toye B. 2004. Suboptimal clinical response to ciprofloxacin in patients with enteric fever due to Salmonella spp. with reduced fluoroquinolone susceptibility: a case series. BMC Infect Dis. 4: 36.

6. Morita M, Hirose K, Takai N, Terajima J, Watanabe H, Sagara H, Kurazono T, Yamaguchi M, Kanazawa Y, Oyaizu T, Izumiya H. 2010. Salmonella enterica serovar Typhi in Japan, 2001-2006: emergence of high-level fluoroquinolone-resistant strains. Epidemiol Infect 138: 318-321.

7. Saha SK, Darmstadt GL, Baqui AH, Crook DW, Islam MN, Islam M, Hossain M, El Arifeen S, Santosham M, Black RE. 2006. Molecular basis of resistance displayed by highly ciprofloxacin-resistant Salmonella enterica serovar Typhi in Bangladesh. J Clin Microbiol. 44: 3811-3813.

8. Yanagi D, de Vries GC, Rahardjo D, Alimsardjono L, Wasito EB, De I., Kinoshita S, Hayashi Y, Hotta H, Osawa R, Kawabata M, Shirakawa T. 2009. Emergence of fluoroquinolone-resistant strains of Salmonella enterica in Surabaya, Indonesia. Diagn Microbiol Infect Dis. 64: 422-426.

9. Asna SM, Haq JA, Rahman MM. 2003. Nalidixic acid-resistant Salmonella enterica serovar Typhi with decreased susceptibility to ciprofloxacin caused treatment failure: a report from Bangladesh. Jpn J Infect. Dis. 56: 32-33.

10. Ahmed D, D'Costa LT, Alam K, Nair GB, Hossain MA. 2006. Multidrug-resistant Salmonella enterica serovar typhi isolates with high-level resistance to ciprofloxacin in Dhaka, Bangladesh. Antimicrob Agents Chemother. 50: 3516-3517.

11. Shirakawa T, Acharya B, Kinoshita S, Kumagai S, Gotoh A, Kawabata M. 2006. Decreased susceptibility to fluoroquinolones and gyrA gene mutation in the Salmonella enterica serovar Typhi and Paratyphi A isolated in Katmandu, Nepal, in 2003. Diagn Microbiol Infect Dis. 54: 299-303.

12. Chau TT, Campbell JI, Galindo CM, Van Minh Hoang N, Diep TS, Nga TT, Van Vinh Chau N, Tuan PQ, Page AL, Ochiai RL, Schultsz C, Wain J, Bhutta ZA, Parry CM, Bhattacharya SK, Dutta S, Agtini M, Dong B, Honghui Y, Anh DD, Canh do G, Naheed A, Albert MJ, Phetsouvanh R, Newton PN, Basnyat B, Arjyal A, La TT, Rang NN, Phuong le T, Van Be Bay P, von Seidlein L, Dougan G, Clemens JD, Vinh H, Hien TT, Chinh NT, Acosta CJ, Farrar J, Dolecek C. 2007. Antimicrobial drug resistance of Salmonella enterica serovar typhi in asia and molecular mechanism of reduced susceptibility to the fluoroquinolones. Antimicrob Agents Chemother. 51: 4315-4323. 
13. Chuang CH, Su LH, Perera J, Carlos C, Tan BH, Kumarasinghe G, So T, Van PH, Chongthaleong A, Hsueh PR, Liu JW, Song JH, Chiu CH. 2009. Surveillance of antimicrobial resistance of Salmonella enterica serotype Typhi in seven Asian countries. Epidemiol Infect. 137: 266-269.

14. Dimitrov T, Dashti AA, Albaksami O, Udo EE, Jadaon MM, Albert MJ. 2009. Ciprofloxacin-resistant Salmonella enterica serovar typhi from Kuwait with novel mutations in $g y r \mathrm{~A}$ and $\operatorname{parC}$ genes. $J$ Clin Microbiol. 47: 208-211.

15. Ouabdesselam S, Tankovic J, Soussy CJ. 1996. Quinolone resistance mutations in the gyrA gene of clinical isolates of Salmonella. Microb. Drug Resist. 2: 299-302.

16. Piddock LJ, Ricci V, McLaren I, Griggs DJ. 1998. Role of mutation in the gyrA and $\operatorname{par} \mathrm{C}$ genes of nalidixic-acid-resistant Salmonella serotypes isolated from animals in the United Kingdom. $J$. Antimicrob. Chemother. 41: 635-641.

17. Turner AK, Nair S, Wain J. 2006. The acquisition of full fluoroquinolone resistance in Salmonella typhi by accumulation of point mutations in the topoisomerase targets. J Antimicrob Chemother. 58: 733-740.

18. Threlfall EJ, Ward LR, Skinner JA, Smith HR, Lacey S. 1999. Ciprofloxacin-resistant Salmonella typhi and treatment failure. Lancet 353: 1590-1591.

19. Saha SK, Khan WA, Saha S.1992. Blood cultures from Bangladeshi children with septicaemia: an evaluation of conventional, lysis-direct plating and lysis-centrifugation methods. Trans R Soc Trop Med Hyg. 86: $554-556$.

20. Cheesebrough M. 1998. Medical laboratory manual for tropical countries. Tropical health technology and butterworth-Heinemann, London, United Kingdom.

21. CLSI. 2006. Performance standards for antimicrobial susceptibility testing. CLSI Document M100-S16. CLSI, Wayne, PA.
22. Liu Y, Lee MA, Ooi EE, Mavis Y, Tan AL, Quek HH. 2003. Molecular typing of Salmonella enterica serovar typhi isolates from various countries in Asia by a multiplex PCR assay on variable-number tandem repeats. J ClinMicrobiol. 41: 4388-4394.

23. Hirose K, Tamura K, Watanabe H. 2003. Screening method for Salmonella enterica serovar Typhi and serovar Paratyphi A with reduced susceptibility to fluoroquinolones by PCR-restriction fragment length polymorphism. Microbiol Immunol. 47: 161-165.

24. Saha SK, Baqui AH, Hanif M, Darmstadt GL, Ruhulamin M, Nagatake T, Santosham M, Black RE. 2001. Typhoid fever in Bangladesh: implications for vaccination policy. Pediatr Infect Dis J. 20: 521524.

25. Sinha A, Sazawal S, Kumar R, Sood S, Reddaiah VP, Singh B, Rao M, Naficy A, Clemens JD, Bhan MK. 1999. Typhoid fever in children aged less than 5 years. Lancet 354: 734-737.

26. Mahle WT, Levine MM. 1993. Salmonella typhi infection in children younger than five years of age. Pediatr Infect Dis J. 12: 627-631.

27. Brooks WA, Hossain A, Goswami D, Nahar K, Alam K, Ahmed N, Naheed A, Nair GB, Luby S, Breiman RF. 2005. Bacteremic typhoid fever in children in an urban slum, Bangladesh. Emerg Infect Dis. 11: 326-329.

28. Brown NM, Millar MR, Frost JA, Rowe B. 1994. Ciprofloxacin resistance in Salmonella paratyphi A. J Antimicrob Chemother. 33: 1258-1259.

29. Chitnis S, Chitnis V, Hemvani N, Chitnis DS. 2006. Ciprofloxacin therapy for typhoid fever needs reconsideration. $J$ Infect Chemother. 12: 402-404.

30. Hakanen AJ, Lindgren M, Huovinen P, Jalava J, Siitonen A, Kotilainen P. 2005. New quinolone resistance phenomenon in Salmonella enterica: nalidixic acid-susceptible isolates with reduced fluoroquinolone susceptibility. J Clin Microbiol. 43: 5775-5778. 